\title{
Identification and differential expression analysis of anthocyanin biosynthetic genes in leaf color variants of ornamental kale
}

\author{
Ning Guo ${ }^{1}$, Shuo Han ${ }^{1}$, Mei Zong ${ }^{1}$, Guixiang Wang ${ }^{1}$, Shuning Zheng ${ }^{2}$ and Fan Liu*
}

\begin{abstract}
Background: Anthocyanins perform diverse biological functions in plants and are beneficial to human health. Leaf color is the most important trait of ornamental kale and the characteristics of changes in leaf color make it an ideal material to elucidate genetic mechanisms of anthocyanins accumulation in Brassica oleracea. To elucidate the anthocyanin distribution, metabolic profiles and differentially expressed anthocyanin biosynthetic genes between different colored accessions can pave the way for understanding the genetic regulatory mechanisms of anthocyanin biosynthesis and accumulation in ornamental kale.

Results: In this study, anthocyanin distributions in red- and white-leaved ornamental kale accessions were determined. Thirty-four anthocyanins were detected in the red-leaved accession. The complete set of anthocyanin biosynthetic genes in the B. oleracea reference genome was identified and differential expression analysis based on RNA-seq was conducted. Eighty-one anthocyanin biosynthetic genes were identified in the B. oleracea reference genome. The expression patterns and differential expressions of these genes in different leaf types indicated that late biosynthetic genes (BODFR1, BOANS1 and 2, and BOUGT79B1.1), positive regulatory genes (BOTTG1, BOTT8, and Bol012528), a negative regulatory gene (BOMYBL2.1), and transport genes (BOTT19.1 and BOTT19.2) may play roles in anthocyanin accumulation in ornamental kale. A genetic regulatory network of anthocyanin accumulation in ornamental kale was constructed.

Conclusions: The distribution of pigments and anthocyanin profiles explained the leaf color phenotypes of ornamental kales. The identification of key genes and construction of genetic regulatory network in anthocyanin accumulation in ornamental kale elucidated the genetic basis of leaf color variants. These findings enhance the understanding of the genetic mechanisms and regulatory network of anthocyanin accumulation in B. oleracea, and provide a theoretical basis for breeding new cultivars of Brassica vegetables with enhanced ornamental and nutritional value.
\end{abstract}

Keywords: Anthocyanin accumulation, Anthocyanin biosynthetic genes, Brassica oleracea, Color variants, Differentially expressed genes, Ornamental kale, Regulatory network

\section{Background}

The colors of leaves, flowers and fruits of agricultural products are highly important for their commercial value. Anthocyanins, which are water-soluble pigments synthesized in many plants, are an important type of flavonoid compound that contribute to the majority of

\footnotetext{
* Correspondence: liufan@nercv.org

'Beijing Vegetable Research Center, Beijing Academy of Agriculture and Forestry Sciences, National Engineering Research Center for Vegetables, Key Laboratory of Biology and Genetic Improvement of Horticultural Crops (North China), Ministry of Agriculture, P. R. China, Beijing 100097, China Full list of author information is available at the end of the article
}

the orange, red, blue, and purple colors. Anthocyanins perform extremely diverse functions in plants. They serve as attractants of pollinators and seed dispersers, and play important roles in responses to abiotic and biotic stresses [33]. Anthocyanins also have beneficial roles in human health. As potent antioxidants, they are potentially protective against cardiovascular disease, certain cancers, and some other chronic diseases [4, 13, 21]. Therefore, a comprehensive understanding of anthocyanin biosynthesis is important for development of foods that are rich in anthocyanins to meet the

(c) The Author(s). 2019 Open Access This article is distributed under the terms of the Creative Commons Attribution 4.0 International License (http://creativecommons.org/licenses/by/4.0/), which permits unrestricted use, distribution, and 
increasing demand for health-promoting components in our daily diet.

The biosynthetic pathways of anthocyanins have been well characterized [12] and the corresponding genes have been isolated from various plant species. In the model plant Arabidopsis thaliana, the biosynthesis, regulation, and transport of anthocyanins, specifically the majority of the structural and regulatory genes involved in anthocyanin synthesis, have been identified and functionally characterized in the last two decades [3, $16,38]$. These studies have made important contributions to the comprehensive understanding of anthocyanin biosynthesis and have revealed the accumulation and metabolic profiles of anthocyanins in Arabidopsis.

Anthocyanins are derived from branches of the flavonoid pathway, which starts with phenylalanine via the general phenylpropanoid pathway. The phenylpropanoid pathway contains three major genes: $P A L$ (phenylalanine ammonia-lyase), $C 4 H$ (cinnamate-4-hydroxylase) and $4 C L$ (4-coumarate: CoA ligase). Two types of correlated structural genes can be distinguished in the flavonoid biosynthetic pathway: early biosynthetic genes (EBGs) and late biosynthetic genes (LBGs) [26]. The EBGs, which include $\mathrm{CHS}$ (chalcone synthase), $\mathrm{CHI}$ (chalcone isomerase), $\mathrm{FHH}$ (flavanone 3-hydroxylase), $F 3^{\prime} H$ (flavonoid 3'-hydroxylase), and FLS (flavonol synthase), lead to the production of flavonols and other flavonoid compounds, whereas the LBGs, which include DFR (dihydroflavonol-4-reductase), ANS (anthocyanidin synthase), and UFGT (UDP-glucose: flavonoid 3-O-glucosyltransferase), lead to the production of anthocyanins [16].

At the transcription level anthocyanin biosynthesis is mainly regulated by a series of transcription factors, especially members of the R2R3-MYB gene family. While EBGs are activated by co-activator-independent and functionally redundant R2R3-MYB genes (e.g., MYB11, MYB12, and MYB111 in Arabidopsis), LBGs are activated by a highly conserved MYB-bHLH-WD40 $(\mathrm{MBW})$ transcriptional activation complex ([11, 26, 41]; $\mathrm{Xu}$ et al., 2015 [40]). In Arabidopsis, the R2R3-MYB genes PAP1, PAP2, MYB113, and MYB114 [2, 8], the bHLH gene family members TT8, GL3, and EGL3 [23, 25, 42], and the WD40 family gene TTG1 [37] are recognized as the key genes encoding respective components of the MBW complex. In addition, two single-repeat R3MYB transcription factors, MYBL2 ([6]a; [22]) and CPC (CAPRICE) [43], and three members of the LATERAL ORGAN BOUNDARY DOMAIN (LBD) gene family, $L B D 37, L B D 38$, and $L B D 39$ [29], are negative regulators of anthocyanin biosynthesis in Arabidopsis.

Anthocyanins are synthesized on the cytosolic surface of the endoplasmic reticulum (ER) but predominantly accumulate in the vacuole. Transparent Testa 19 (TT19), a glutathione S-transferase (GST), which functions as a carrier to transport anthocyanins to the tonoplast, plays a key role in anthocyanins transportation and accumulation. TT19 binds to the anthocyanins synthesized on the cytosolic surface of the ER. The pigment-TT19 binary complex in the cytosol is recruited to the tonoplast. The pigments may be acylated, prior to release from TT19, and subsequently sequestered into vacuoles by transporters [32].

Numerous vegetable crops derived from Brassica oleracea are important worldwide, including cauliflower, broccoli, cabbage, Brussels sprouts, kohlrabi, and kale. Almost all cultivar groups include red- or purplepigmented types, in which anthocyanins are deposited in various organs, especially the leaves, stems, siliques, and curds [27]. Ornamental kale (B. oleracea var. acephala), which is an excellent ornamental foliage plant with a range of leaf colors and shapes, is widely cultivated as a landscape plant, potted plant, and cut flower. It is sufficiently tolerant of frost and chilling that it can grow vigorously in regions that experience low temperature [17]. The variety of leaf colors is the most typical characteristic of ornamental kale. The outer, mature leaves are green, whereas the central (newly developed) leaves may be white, pink, red, or purple. The color of the new leaves is determined by the content of anthocyanins. Leaf color is the most important trait of kale for its ornamental value and nutritional quality, as well as cold resistance.

Some leaf color-related candidate genes have been mapped in ornamental kale. For example, the red leaf color trait is reportedly controlled by the single dominant gene $R e$ in ornamental kale, which was mapped to chromosome C09 [28]. Zhu et al. determined the pink leaf color trait in ornamental kale to be controlled by a single semi-dominant gene mapped to chromosome $\mathrm{C} 03$ [44]. $B o P r$, which was identified as a gene encoding a dihydroflavonol reductase (DFR), was mapped on chromosome C09 and controls the purple leaf trait of ornamental kale [20]. The change in color of the inner leaves (from green to red or white) of ornamental kales is induced by low temperature, and thus may be a type of low temperature adaptation. The mechanism underlying such color formation remains poorly understood. A complete understanding of the structural and regulatory genes involved as well as analysis of the differential expression patterns in young and mature leaves and among cultivars is important for elucidation of the mechanism of anthocyanin biosynthesis and leaf pigmentation in ornamental kale.

In a previous study, the anthocyanin biosynthetic genes in B. rapa (BrABGs) were identified and analyzed by comparative genomic analysis [9]. In the present study, the anthocyanin profile was determined in red ornamental kale using liquid chromatography-mass 
spectrometry (LC-MS), the complete set of anthocyanin biosynthetic genes in B. oleracea was identified by comparative genomic analysis, and RNA sequencing (RNAseq) of central (new) and outer (mature) leaves from red- and white-leaved ornamental kales was conducted to analyze the differential expression of anthocyanin biosynthetic genes. The results of our studies will advance the understanding of the mechanism of anthocyanin biosynthesis at the gene expression level, and provide a foundation for further cultivar improvement and the breeding of novel cultivars of enhanced ornamental and nutritional value.

\section{Results}

\section{Anthocyanin distribution in red- and white-leaved} ornamental kales

We observed that the ornamental kales began to show color development, i.e. the newly developing leaves became red or white, when the minimum temperature decreased below $10^{\circ} \mathrm{C}$. For the red-leaved $\mathrm{DH}$ line '05-DH65, the central new leaves are purple-red (Fig. 1c), the petiole and veins of the outer, mature leaves are red, whereas the remainder of the leaf lamina is green (Fig. 1d) . For the white-leaved DH line '06-DH-71', the central new leaves are almost white (Fig. 1e) and the outer mature leaves are green (Fig. 1f). Leaf anatomical observations revealed that in the lamina of new leaves of '05-DH-65', red-purple pigmentation was present in the adaxial and abaxial epidermal cells as well as one to several adjacent cell layers of the palisade or spongy mesophyll, whereas internal mesophyll cells were transparent and contained almost no pigments, including chlorophylls (Fig. 1g). The transverse and longitudinal sections of the veins and petiole of new leaves of '05-DH-65' revealed an identical distribution of pigments to that of the lamina. With regard to mature leaves of '05-DH-65', the distribution of redpurple pigments was identical to that of new leaves; however, red-purple pigments were less abundant in mature leaves and the internal leaf mesophyll cells, except in the veins and petioles, contained chlorophylls and appeared green, while the veins and petiole were red-purple (Fig. 1d, h, l, p). Sections of new leaves of '06-DH-71' revealed that almost no pigments were present in all cells (Fig. 1I, m, q). Mature leaves of '06-DH-71' contained chlorophylls but no red pigments (Fig. 1j, n, r).

\section{Anthocyanins profile and content analysis}

The red-purple pigments in ornamental kale are due to anthocyanins. To characterize the anthocyanin profile, we performed UHPLC-TOF analysis of new leaves of the redleaved DH line '05-DH-65'. In total, 34 anthocyanin compounds were detected (Fig. 2a; Table 1). Identification of anthocyanins in extracts was based on a method combining chromatographic behavior, accurate molecular mass spectra obtained by Q-TOF mass spectrometry, characteristic MS/MS fragmentation product ions obtained by QTrip mass spectrometry, UV spectra, and comparison with previous publications $[10,18,39]$. The glycosyl groups were hexoses, which were mainly glucosides, and ranged in the number from one to four. The glycosyl groups were acylated by sinapoyl, $p$-coumaroyl, caffeoyl, feruloyl, and hydroxyferuloyl. Several isomers with an identical formula were detected, thus the glycosyl groups may be located in different positions. A comprehensive metabolic profile of anthocyanins in '05-DH-65' was generated (Table 1). The TAC in new and mature leaves of '05-DH-65' and '06-DH-71' were determined by $\mathrm{pH}$ differential spectrophotometry. The TACs in new and mature leaves of '05-DH-65' were 11.04 and $0.54 \mathrm{mg} /$ g DW, respectively, whereas the TACs in new and mature leaves of '06-DH-71' were 0.073 and $0 \mathrm{mg} / \mathrm{g}$ DW, respectively (Fig. 2b).

\section{Identification, chromosomal localization and subgenomic distribution of anthocyanin biosynthetic genes}

Forty-one anthocyanin biosynthetic genes (ABGs) have been identified in the Arabidopsis genome, consisting of 24 structural genes encoding anthocyanin biosynthetic enzymes, 16 regulatory genes encoding transcriptional factors, and one transport gene that is required for anthocyanin transportation (Table 2). Based on a combination of syntenic and non-syntenic homology analysis, 81 B. oleracea anthocyanin biosynthetic genes (BoABGs) were identified, representing homologs for 39 of the 41 AtABGs; homologs of two AtABGs (AtFLS6 and AtMYB11) were not detected in B. oleracea. Among the 81 BoABGs, 57 were syntenic orthologs and 24 were non-syntenic homologs. The major events responsible for BoABGs copy number expansion were whole genome duplication (WGD) and tandem duplication (TD). Nine AtABGs had two syntenic orthologs and an additional nine AtABGs had three syntenic orthlogs. The BoABGs had less than three syntenic orthologs as a result of gene fractionation that occurred following the WGT. Some AtABGs were TD genes that formed a gene cluster, such as AtFSL2-5, four FSL-encoding genes, and three R2R3-MYB genes (AtPAP1, AtMYB113, and AtMYB114) that form the MBW complex to regulate LBGs; their syntenic $B$. oleracea orthologs had both lost the TD paralogs. However, some BoABGs, especially structural genes, had undergone TD to form gene clusters, such as Bo4CL5.1 to 5.5, BoF3H4 and BoF3H5, BoFLS1.2 and BoFLS1.3, and BoUGT79B1.2 to 1.5. These TDs were all novel in $B$. oleracea, which represented an important means to increase gene number. For example, syntenic orthologs of At4CL5 were lacking in B. oleracea, whereas non-syntenic orthologs formed a tandem cluster of five genes. The same pattern was observed for 


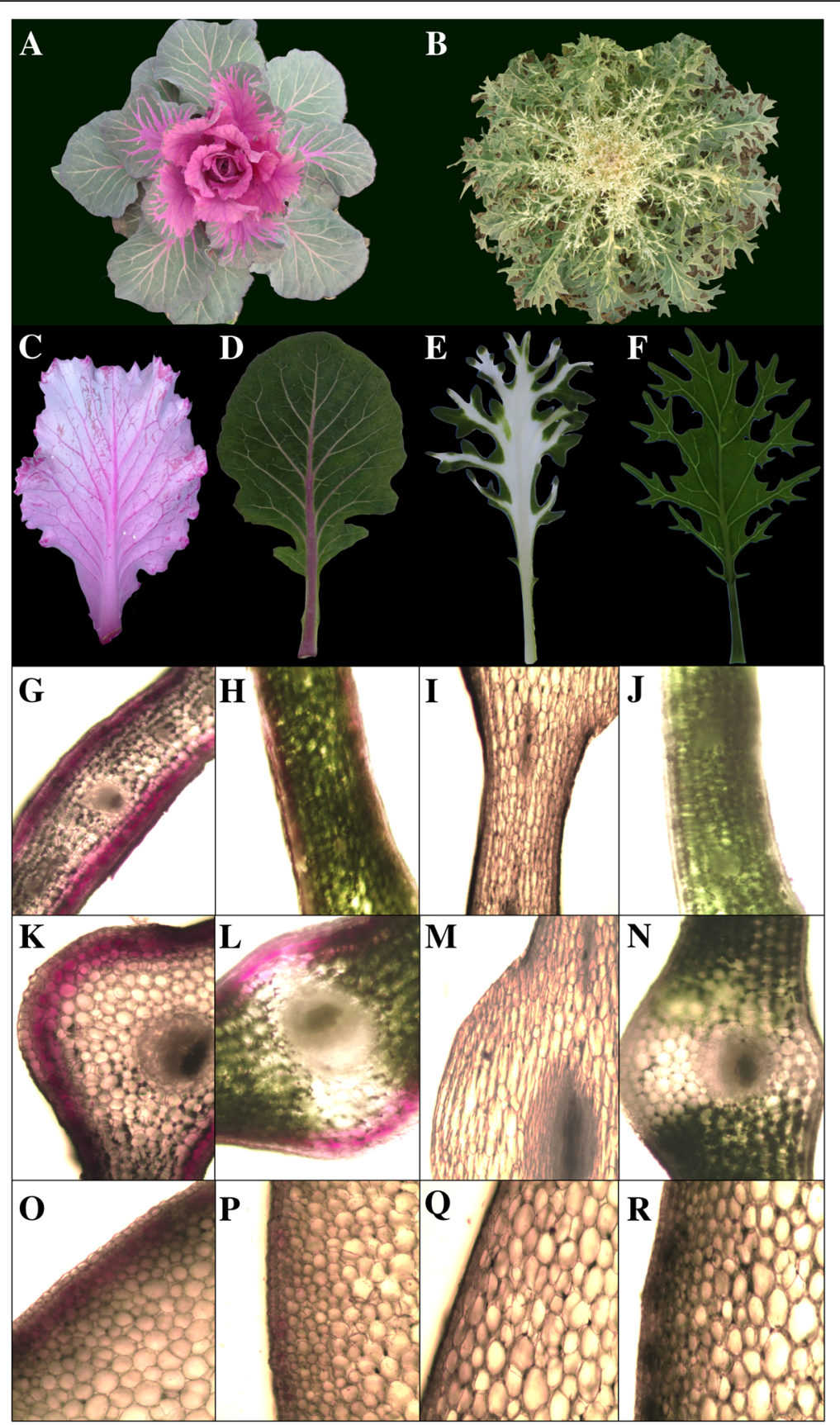

Fig. 1 Individual plants, new and mature leaves, and anatomical distribution of leaf pigments in ornamental kale $\mathrm{DH}$ lines '05-DH-65' and '06-DH71'. (a) '05-DH-65' plant; (b) '06-DH-71' plant; (c) '05-DH-65' new (central) leaf; (d) '05-DH-65' mature (outer) leaf; (e) '06-DH-71' new (central) leaf; (f) '06-DH-71' mature (outer) leaf; (g) '05-DH-65' new leaf lamina, transverse section; (h) '05-DH-65' mature leaf lamina, transverse section; (i) '06$\mathrm{DH}-71$ ' new leaf lamina, transverse section; (j) '06-DH-71' mature leaf lamina, transverse section; (k) '05-DH-65' new leaf vein, transverse section; (I) '05-DH-65' mature leaf vein, transverse section; $(\mathbf{m})$ '06-DH-71' new leaf vein, transverse section; (n) '06-DH-71' mature leaf vein, transverse section; (o) '05-DH-65' new leaf petiole, longitudinal section; (p) '05-DH-65' mature leaf petiole, longitudinal section; (q) '06-DH-71' new leaf petiole, longitudinal section; (r) '06-DH-71' mature leaf petiole, longitudinal section

AtUGT79B1, which did not experience WGD but its non-syntenic orthologs were duplicated by TD.

Of the 81 BoABGs, 56 were mapped to the nine chromosomes of B. oleracea. Thus, 2, 7, 8, 7, 5, 9, 5, 7, and 6 BoABGs were located on chromosomes C01 to C09, respectively, of $B$. oleracea genome V1.1 (Cap 02-12) (Fig. 3a). A higher number of BoABGs (73) were mapped on the genome V2.1 (TO1000) [24] (Fig. 3b), with 3, 6, $10,14,5,8,7,7$, and 13 located on chromosomes C01 to $\mathrm{C} 09$, respectively. However, the distributions of the 

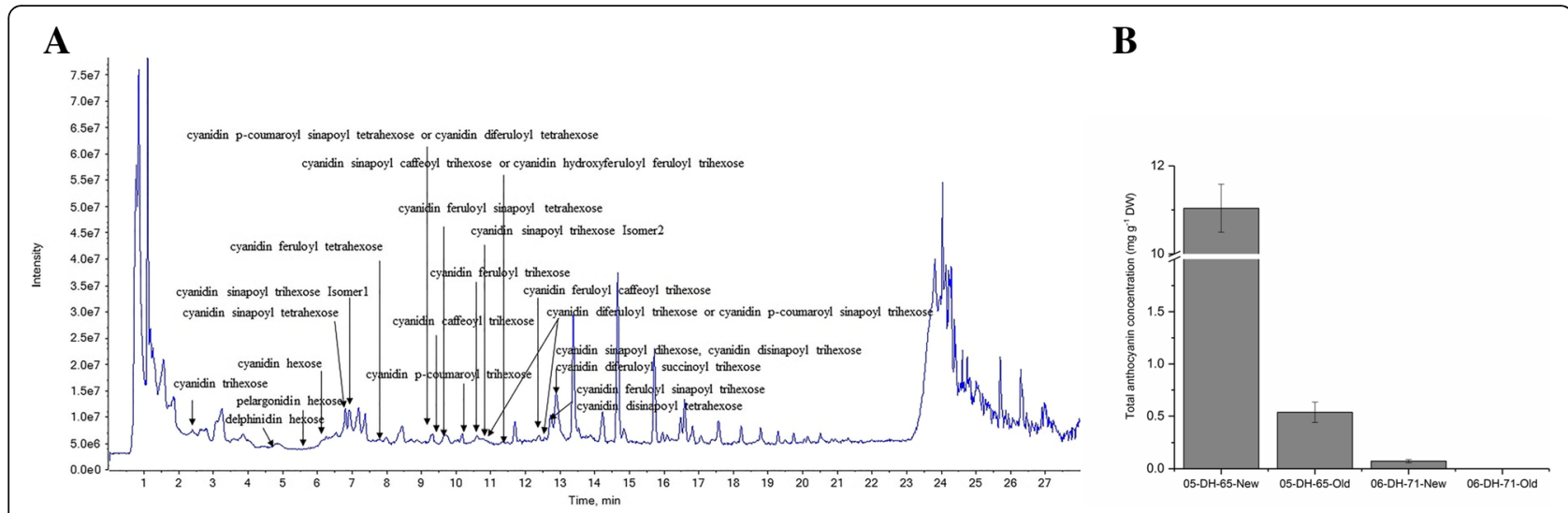

Fig. 2 a Liquid chromatography chromatograms $(520 \mathrm{~nm}$ ) of anthocyanin extracts from new leaves of the ornamental kale DH line '05-DH-65'. Horizontal axis shows retention time (min); vertical axis shows the strength of the chromatographic peak response intensity. The compound name is provided for each peak. $\mathbf{b}$ Total anthocyanins content in new and mature leaves of the DH lines '05-DH-65' and '06-DH-71'

genes differed between the two reference genome versions. The corresponding gene IDs of ABGs in the two genome versions are listed in Additional file 7: Table S1.

\section{Overall expression of genes in the anthocyanin biosynthesis pathway}

From mRNA sequencing, raw data were obtained from three biological repeats for new and mature leaves of the red- and white-leaved DH lines '05-DH-65' and '06-DH-71'. The summary statistics of the RNA sequencing was showed in Additional file 7: Table S2. Gene expression levels were calculated using the expected FPKM values. The expression values distribution of different samples were showed in Additional file 1: Figure S1A which demonstrated the overall expression levels among different samples. In order to validate these biological replicates sampled with good agreement replicates, these samples were clustered by gene expression values between each other (Additional file 1: Figure S1B). From these results we could clearly see that the three biological repeats were clustered together indicating good repeatability.

Of the 81 BoABGs, expression of 15 genes was not detected and six genes showed extremely low expression levels (FPKM value $<1$ ) in all of the new and mature leaves of '05DH-65' and '06-DH-71'. These genes were identified as duplicated paralogs that arose by WGD or TD, and at least one of the paralogs was expressed. For example, of the five identified flavanone 3-hydroxylase (F3H) coding genes, BoF3H1 to 5, only BoF3H1 was expressed in new and mature leaves of '05-DH-65' and '06-DH-71', whereas expression of the other four paralogs was not detected. BoDFR2 was not expressed in each of the four leaf types, whereas its paralog BoDFR1 was differentially expressed in new and mature leaves of the two accessions. Some genes were especially expressed in '05DH-65', such as Bo4CL2.1 and BoUGT79B1.1, whereas the negative regulatory gene BoMYBL2.1 was only expressed in '06-DH-71'. The expression FPKM values are listed in Additional file 7: Table S3.

To determine the expression patterns of BoABGs, a heatmap combined with a hierarchical clustering analysis was constructed based on gene expression (FPKM values were $\log 2$ transformed) levels in new and mature leaves of '05DH-65' and '06-DH-71'. The expression levels of BoABGs varied among the different leaves (Additional file 2: Figure S2). Anthocyanin biosynthetic genes from new and mature leaves of the same accession were clustered in the same group based on the BoABGs expression levels. Thus, BoABGs showed different expression patterns in the redand white-leaved accessions. The 81 BoABGs were clustered into two main groups. The first group contained five genes (BoCHS1, 2, 3, BoF3H1, and BoFLS1.1) that were expressed at relatively higher levels in all of the four leaf types. These genes were all EBGs that lead to the production of flavonols and other flavonoid compounds. The second group comprised two subgroups. In the first subgroup were the genes expressed at low levels in almost all of the four leaf types. As already mentioned, these genes were mainly paralogs that were expanded by WGD or TD. The genes in the second subgroup showed differential expression in the four leaf types. BoDFR1, BoANS1, BoANS2, BoUGT75C1, and BoUGT79B1, which are all LBGs, were most highly expressed in new leaves of '05-DH-65' and showed the lowest expression level in mature leaves of '06-DH-71'. The transport genes BoTT19.1 and BoTT19.2 showed an identical expression pattern.

\section{The expression analysis of genes in the anthocyanin biosynthetic pathway among different leaf types} The variation in expression of structural and regulatory genes is illustrated in Fig. 4, in which the duplicated 
Table 1 UHPLC-PDA-Q-TOF data and putative identification of anthocyanins from new leaves of the ornamental kale DH line '05DH-65'

\begin{tabular}{|c|c|c|c|c|}
\hline Anthocyanins & Formula & $\begin{array}{l}t_{R} \\
(\min )^{a}\end{array}$ & $\begin{array}{l}\text { Mass } \\
(\mathrm{Da})\end{array}$ & $\begin{array}{l}\text { Major and important } \\
\text { productions }(\mathrm{m} / \mathrm{z})\end{array}$ \\
\hline Cyanidin hexose Isomer1 & $\mathrm{C} 21 \mathrm{H} 21 \mathrm{O} 11$ & 6.16 & 449.11 & 287.06 \\
\hline Cyanidin hexose Isomer2 & $\mathrm{C} 21 \mathrm{H} 21 \mathrm{O} 11$ & 7.37 & 449.11 & 287.06 \\
\hline Cyanidin hexose Isomer3 & $\mathrm{C} 21 \mathrm{H} 21 \mathrm{O} 11$ & 12.37 & 449.11 & 287.05 \\
\hline Cyanidin hexose Isomer4 & $\mathrm{C} 21 \mathrm{H} 21 \mathrm{O} 11$ & 12.57 & 449.11 & 287.06 \\
\hline Cyanidin dihexose Isomer1 & $\mathrm{C} 27 \mathrm{H} 31 \mathrm{O} 16$ & 6.12 & 611.16 & $449.10,287.05$ \\
\hline Cyanidin dihexose Isomer2 & $\mathrm{C} 27 \mathrm{H} 31 \mathrm{O} 16$ & 6.35 & 611.16 & 287.05 \\
\hline Cyanidin trihexose Isomer1 & $\mathrm{C} 33 \mathrm{H} 41 \mathrm{O} 21$ & 2.46 & 773.21 & $611.16,449.11,287.06$ \\
\hline Cyanidin trihexose Isomer2 & $\mathrm{C} 33 \mathrm{H} 41 \mathrm{O} 21$ & 6.11 & 773.21 & $611.16,449.11,287.05$ \\
\hline Cyanidin sinapoyl dihexose & $\mathrm{C} 38 \mathrm{H} 41 \mathrm{O} 20$ & 12.90 & 817.22 & $655.16,499.11,287.06$ \\
\hline Cyanidin p-coumaroyl trihexose & $\mathrm{C} 42 \mathrm{H} 47 \mathrm{O} 23$ & 10.24 & 919.25 & $757.19,49.11,287.05$ \\
\hline Cyanidin caffeoyl trihexose & $\mathrm{C} 42 \mathrm{H} 47 \mathrm{O} 24$ & 9.17 & 935.25 & $773.20,449.11,287.05$ \\
\hline Cyanidin tetrahexose Isomer 1 & $\mathrm{C} 39 \mathrm{H} 51 \mathrm{O} 26$ & 6.35 & 935.27 & $773.21,611.16,449.11,287.05$ \\
\hline Cyanidin tetrahexose Isomer2 & $\mathrm{C} 39 \mathrm{H} 51 \mathrm{O} 26$ & 7.47 & 935.27 & $773.22,611.16,449.11,287.06$ \\
\hline Cyanidin feruloyl trihexose & $\mathrm{C} 43 \mathrm{H} 49 \mathrm{O} 24$ & 10.65 & 949.26 & $787.21,449.11,287.06$ \\
\hline Cyanidin hydroxyferuloyl trihexose & $\mathrm{C} 43 \mathrm{H} 49 \mathrm{O} 25$ & 6.47 & 965.26 & $803.21,449.11,287.05$ \\
\hline Cyanidin sinapoyl trihexose Isomer1 & $\mathrm{C} 44 \mathrm{H} 51 \mathrm{O} 25$ & 6.92 & 979.27 & $817.22,449.11,287.06$ \\
\hline Cyanidin sinapoyl trihexose Isomer2 & $\mathrm{C} 44 \mathrm{H} 51 \mathrm{O} 25$ & 10.81 & 979.27 & $817.22,449.11,287.06$ \\
\hline Cyanidin feruloyl tetrahexose & $\mathrm{C} 49 \mathrm{H} 59 \mathrm{O} 29$ & 7.75 & 1111.31 & $949.26,449.11,287.05$ \\
\hline Cyanidin feruloyl caffeoyl trihexose & $\mathrm{C} 52 \mathrm{H} 55 \mathrm{O} 27$ & 12.42 & 1111.29 & $949.24,449.11,287.05$ \\
\hline $\begin{array}{l}\text { Cyanidin diferuloyl trihexose or Cyanidin p-coumaroyl } \\
\text { sinapoyl trihexose Isomer1 }\end{array}$ & $\mathrm{C} 53 \mathrm{H} 57 \mathrm{O} 27$ & 10.73 & 1125.31 & $963.24,449.11,287.05$ \\
\hline $\begin{array}{l}\text { Cyanidin diferuloyl trihexose or Cyanidin p-coumaroyl } \\
\text { sinapoyl trihexose Isomer2 }\end{array}$ & $\mathrm{C} 53 \mathrm{H} 57 \mathrm{O} 27$ & 12.39 & 1125.31 & $963.26,449.11,287.06$ \\
\hline Cyanidin sinapoyl tetrahexose & $\mathrm{C} 50 \mathrm{H} 61030$ & 6.91 & 1141.32 & $979.27,817.21,449.11,287.05$ \\
\hline $\begin{array}{l}\text { Cyanidin sinapoyl caffeoyl trihexose or Cyanidin hydroxyferuloyl } \\
\text { feruloyl trihexose }\end{array}$ & $\mathrm{C} 53 \mathrm{H} 57 \mathrm{O} 28$ & 11.29 & 1141.30 & $979.25,449.11,287.06$ \\
\hline Cyanidin feruloyl sinapoyl trihexose & $\mathrm{C} 54 \mathrm{H} 59 \mathrm{O} 28$ & 12.73 & 1155.32 & $993.27,449.11,287.06$ \\
\hline Cyanidin disinapoyl trihexose & $\mathrm{C} 55 \mathrm{H} 61 \mathrm{O} 29$ & 12.90 & 1185.33 & $1023.28,449.1077 ; 287.0573$ \\
\hline Cyanidin diferuloyl succinoyl trihexose & $\mathrm{C} 57 \mathrm{H} 61030$ & 12.93 & 1225.32 & $1045.27,759.22,287.06$ \\
\hline $\begin{array}{l}\text { Cyanidin p-coumaroyl sinapoyl tetrahexose or Cyanidin diferuloyl } \\
\text { tetrahexose }\end{array}$ & $\mathrm{C} 59 \mathrm{H} 67 \mathrm{O} 32$ & 9.44 & 1287.36 & $1225.31,449.11,287.05$ \\
\hline Cyanidin feruloyl sinapoyl tetrahexose & $\mathrm{C} 60 \mathrm{H} 69 \mathrm{O} 33$ & 9.75 & 1317.37 & $1155.32,449.11,287.06$ \\
\hline Cyanidin disinapoyl tetrahexose & $\mathrm{C} 61 \mathrm{H} 71 \mathrm{O} 34$ & 12.88 & 1347.38 & $1185.33,449.09,287.06$ \\
\hline Pelargonidin hexose & $\mathrm{C} 21 \mathrm{H} 21 \mathrm{O} 10$ & 5.68 & 433.11 & 271.06 \\
\hline Delphinidin hexose Isomer1 & $\mathrm{C} 21 \mathrm{H} 21 \mathrm{O} 12$ & 4.82 & 465.10 & 303.05 \\
\hline Delphinidin hexose Isomer2 & $\mathrm{C} 21 \mathrm{H} 21 \mathrm{O} 12$ & 6.81 & 465.10 & 303.05 \\
\hline Delphinidin hexose Isomer3 & $\mathrm{C} 21 \mathrm{H} 21 \mathrm{O} 12$ & 10.44 & 465.10 & 303.05 \\
\hline Delphinidin hexose Isomer4 & $\mathrm{C} 21 \mathrm{H} 21 \mathrm{O} 12$ & 11.84 & 465.10 & 303.05 \\
\hline
\end{tabular}

${ }^{a} t_{R}$ : retention time

paralogs are listed together. The expression levels of duplicated paralogs were highly variable. Some paralogs showed extremely low expression levels or expression was not detected, which indicated that these duplicated genes showed functional redundancy and only a portion of paralogs play roles in anthocyanin biosynthesis.
Examples are the five $F 3 H$ genes $(B o F 3 H 1-5)$ and the two BoDFRs that have been mentioned previously.

Most of upstream genes (phenylpropanoid pathway genes and EBGs) did not show differential expressions in different leaves, which indicated that these genes did not play important roles for anthocaynin accumulation 
Table 2 Anthocyanin biosynthetic genes identified in B. oleracea by comparative genomic analysis with the Arabidopsis thaliana genome

\begin{tabular}{|c|c|c|c|c|}
\hline \multirow{3}{*}{ A. thaliana } & \multicolumn{4}{|l|}{ B. oleracea } \\
\hline & \multicolumn{3}{|l|}{ Synteny orthologs } & \multirow[t]{2}{*}{ Non-synteny orthologs } \\
\hline & $\mathrm{LF}$ & MF1 & MF2 & \\
\hline \multicolumn{5}{|l|}{ Structural genes } \\
\hline \multicolumn{5}{|c|}{ Biosynthetic genes in phenylpropanoid pathway } \\
\hline AtPAL1 (AT2G37040) & BoPAL1.1 (Bol025522) & BoPAL1.2 (Bol037689) & & \\
\hline AtPAL2 (AT3G53260) & BoPAL2.1 (Bol025102) & BoPAL2.2 (Bol041738) & BoPAL2.3 (Bol005411) & BoPAL2.4 (Bol005084) \\
\hline AtPAL3 (AT5G04230) & - & - & BoPAL3.1 (Bol005493) & BoPAL3.2 (Bol006745) \\
\hline AtPAL4 (AT3G10340) & BoPAL4 (Bol011375) & - & - & - \\
\hline \multirow[t]{2}{*}{ AtC4H (AT2G30490) } & - & BoC4H1 (Bol033347) & BoC4H3 (Bol004608) & BoC4H5 (Bol006704) \\
\hline & & BoC4H2 (Bol033349) & BoC4H4 (Bol004610) & \\
\hline At4CL1 (AT1G51680) & - & - & Bo4CL1 (Bol031583) & - \\
\hline \multirow[t]{2}{*}{ At4CL2 (AT3G21240) } & - & - & - & Bo4CL2.1 (Bol038385) \\
\hline & & & & Bo4CL2.2 (Bol038386) \\
\hline At4CL3 (AT1G65060) & Bo4CL3 (Bol012584) & - & - & - \\
\hline \multirow[t]{5}{*}{ At4CL5 (AT3G21230) } & - & - & - & Bo4CL5.1 (Bol000926) \\
\hline & & & & Bo4CL5.2 (Bol038387) \\
\hline & & & & Bo4CL5.3 (Bol038389) \\
\hline & & & & Bo4CL5.4 (Bol026622) \\
\hline & & & & Bo4CL5.5 (Bol026623) \\
\hline \multicolumn{5}{|l|}{ Early biosynthetic genes } \\
\hline AtCHS (AT5G13930) & BoCHS1 (Bol043396) & BoCHS2 (Bol034259) & BoCHS3 (Bol004244) & \\
\hline AtCHI (AT3G55120) & BoCHI1 (Bol044343) & BoCHI2 (Bol044344) & BoCHI3 (Bol008652) & BoCHI4 (Bol018696) \\
\hline \multirow[t]{2}{*}{ AtF3H (AT3G51240) } & BoF3H1 (Bol010585) & BoF3H2 (Bol030864) & BoF3H3 (Bol002277) & BoF3H4 (Bol044664) \\
\hline & & & & BoF3H5 (Bol041656) \\
\hline AtF3'H (AT5G07990) & BoF3'H (Bol043829) & & & \\
\hline \multirow[t]{2}{*}{ AtFLS1 (AT5G08640) } & BoFLS1.1 (Bol043773) & & & BoFLS1.2 (Bol004505) \\
\hline & & & & BoFLS1.3 (Bol019362) \\
\hline $\begin{array}{l}\text { AtFSL2 (AT5G63580) } \\
\text { AtFLS3 (AT5G63590) }\end{array}$ & & & BoFLS2 (Bol019125) & \\
\hline \multicolumn{5}{|l|}{ AtFLS4 (AT5G63595) } \\
\hline \multicolumn{5}{|l|}{ AtFLS5 (AT5G63600) } \\
\hline \multicolumn{5}{|l|}{ AtFLS6 (AT5G43935) } \\
\hline \multicolumn{5}{|l|}{ Late biosynthetic genes } \\
\hline AtDFR (AT5G42800) & & & BoDFR1 (Bol035269) & BoDFR2 (Bol006005) \\
\hline AtANS (AT4G22880) & BoANS1 (Bol014986) & BoANS2 (Bol042059) & & \\
\hline \multirow[t]{4}{*}{ AtUGT79B1 (AT5G54060) } & $\begin{array}{l}\text { BoUGT79B1.1 } \\
\text { (Bol038805) }\end{array}$ & & & $\begin{array}{l}\text { BoUGT79B1.2 } \\
\text { (Bol014515) }\end{array}$ \\
\hline & & & & $\begin{array}{l}\text { BoUGT79B1.3 } \\
\text { (Bol014517) }\end{array}$ \\
\hline & & & & $\begin{array}{l}\text { BoUGT79B1.4 } \\
\text { (Bol014519) }\end{array}$ \\
\hline & & & & $\begin{array}{l}\text { BoUGT79B1.5 } \\
\text { (Bol018228) }\end{array}$ \\
\hline AtUGT75C1 (AT4G14090) & & & BoUGT75C1 (Bol027055) & \\
\hline AtUGT78D2 (AT5G17050) & & & $\begin{array}{l}\text { BoUGT78D2 } \\
\text { (Bol021317) }\end{array}$ & \\
\hline
\end{tabular}


Table 2 Anthocyanin biosynthetic genes identified in B. oleracea by comparative genomic analysis with the Arabidopsis thaliana genome (Continued)

\begin{tabular}{|c|c|c|c|c|}
\hline \multirow{3}{*}{ A. thaliana } & \multicolumn{4}{|l|}{ B. oleracea } \\
\hline & \multicolumn{3}{|l|}{ Synteny orthologs } & \multirow[t]{2}{*}{ Non-synteny orthologs } \\
\hline & $\overline{\mathrm{LF}}$ & MF1 & MF2 & \\
\hline \multicolumn{5}{|c|}{ Regulatory genes (Transcription factor) } \\
\hline \multicolumn{5}{|l|}{ Positive regulators } \\
\hline \multicolumn{5}{|l|}{ R2R3-MYB } \\
\hline \multicolumn{5}{|c|}{ Independent regulatory genes } \\
\hline \multicolumn{5}{|l|}{ AtMYB11 (AT3G62610) } \\
\hline AtMYB12 (AT2G47460) & BoMYB12.1 (Bol001533) & BoMYB12.2 (Bol002581) & BoMYB12.3 (Bol029626) & \\
\hline AtMYB111 (AT5G49330) & BoMYB111.1 (Bol016599) & $\begin{array}{l}\text { BoMYB111.2 } \\
\text { (Bol033054) }\end{array}$ & $\begin{array}{l}\text { BoMYB111.3 } \\
\text { (Bol032351) }\end{array}$ & \\
\hline \multicolumn{5}{|c|}{ Regulation by forming MBW complex } \\
\hline \multicolumn{5}{|l|}{ AtPAP1 (AT1G56650) } \\
\hline AtPAP2 (AT1G66390) & & & & \multirow{6}{*}{$\begin{array}{l}\text { Bol042409 } \\
\text { Bol012531 }\end{array}$} \\
\hline AtMYB113 (AT1G66370) & Bol012528 & Bol045347 & & \\
\hline \multicolumn{4}{|l|}{ AtMYB114 (AT1G66380) } & \\
\hline \multicolumn{4}{|l|}{ bHLH } & \\
\hline AtTT8 (AT4G09820) & BoTT8 (Bol004077) & & & \\
\hline AtGL3 (AT5G41315) & BoGL3 (Bol014556) & & & \\
\hline AtEGL3 (AT1G63650) & & BoEGL3.1 (Bol022614) & BoEGL3.2 (Bol029662) & BoEGL3.3 (Bol004759) \\
\hline \multicolumn{5}{|l|}{ WD40 } \\
\hline AtTTG1 (AT5G24520) & BoTTG1 (Bol022420) & & & \\
\hline \multicolumn{5}{|l|}{ Negative regulators } \\
\hline \multicolumn{5}{|l|}{ Single-Repeat R3 MYB } \\
\hline AtMYBL2 (AT1G71030) & BoMYBL2.1 (Bol016164) & BoMYBL2.2 (Bol034966) & & \\
\hline AtCPC (AT2G46410) & BoCPC1 (Bol000928) & BoCPC2 (Bol021780) & BoCPC3 (Bol029590) & \\
\hline \multicolumn{5}{|c|}{$\begin{array}{l}\text { LATERAL ORGAN BOUNDARY DOMAIN } \\
\text { (LBD) }\end{array}$} \\
\hline AtLBD37 (AT5G67420) & BoLBD37.1 (Bol014304) & BoLBD37.2 (Bol008082) & BoLBD37.3 (Bol005707) & \\
\hline AtLBD38 (AT3G49940) & BoLBD38.1 (Bol007980) & BoLBD38.2 (Bol021982) & BoLBD38.3 (Bol016975) & \\
\hline AtLBD39 (AT4G37540) & & & & BoLBD39 (Bol019060) \\
\hline \multicolumn{5}{|l|}{ Transport genes } \\
\hline AtTT19 (AT5G17220) & BoTT19.1 (Bol019821) & & BoTT19.2 (Bol021325) & \\
\hline
\end{tabular}

differences. The LBGs, such as BoDFR1, BoANS1, BoANS2 and BoUGT79B1.1, showed differential expression levels in new and mature leaves of '05-DH-65' and '06-DH-71'. These genes all showed the highest expression levels in new leaves of '05-DH-65', second-highest levels in mature leaves of '05-DH-65', and low expression levels in new and mature leaves of '06-DH-71', which are white and green and contain almost no anthocyanins (Fig. 4).

The expression of structural genes in the anthocyanin biosynthetic pathway is mainly regulated by R2R3-MYB, basic helix-loop-helix (bHLH) and WD40-type transcriptional factors and their interaction [14]. Some R2R3-
MYB proteins, such as MYB11, MYB12, and MYB111, which independently regulate EBGs [31], showed relatively low expression levels in the four leaf types and mostly did not show significant differences in expression level; only BoMYB111.1 was more highly expressed in '06-DH-71' leaves than in '05-DH-65' leaves (Fig. 4). The LBGs are mainly activated by MBW ternary transcriptional complexes [8]. Of the four R2R3-MYB genes (Bol012528, Bol012531, Bol045347, and Bol042409) that form the MBW complex, Bol012531 showed extremely low expression levels in all four leaf types; Bol042409 showed a higher expression level in '06-DH-71' leaves than in '05-DH-65' leaves; Bol045347 showed the lowest 


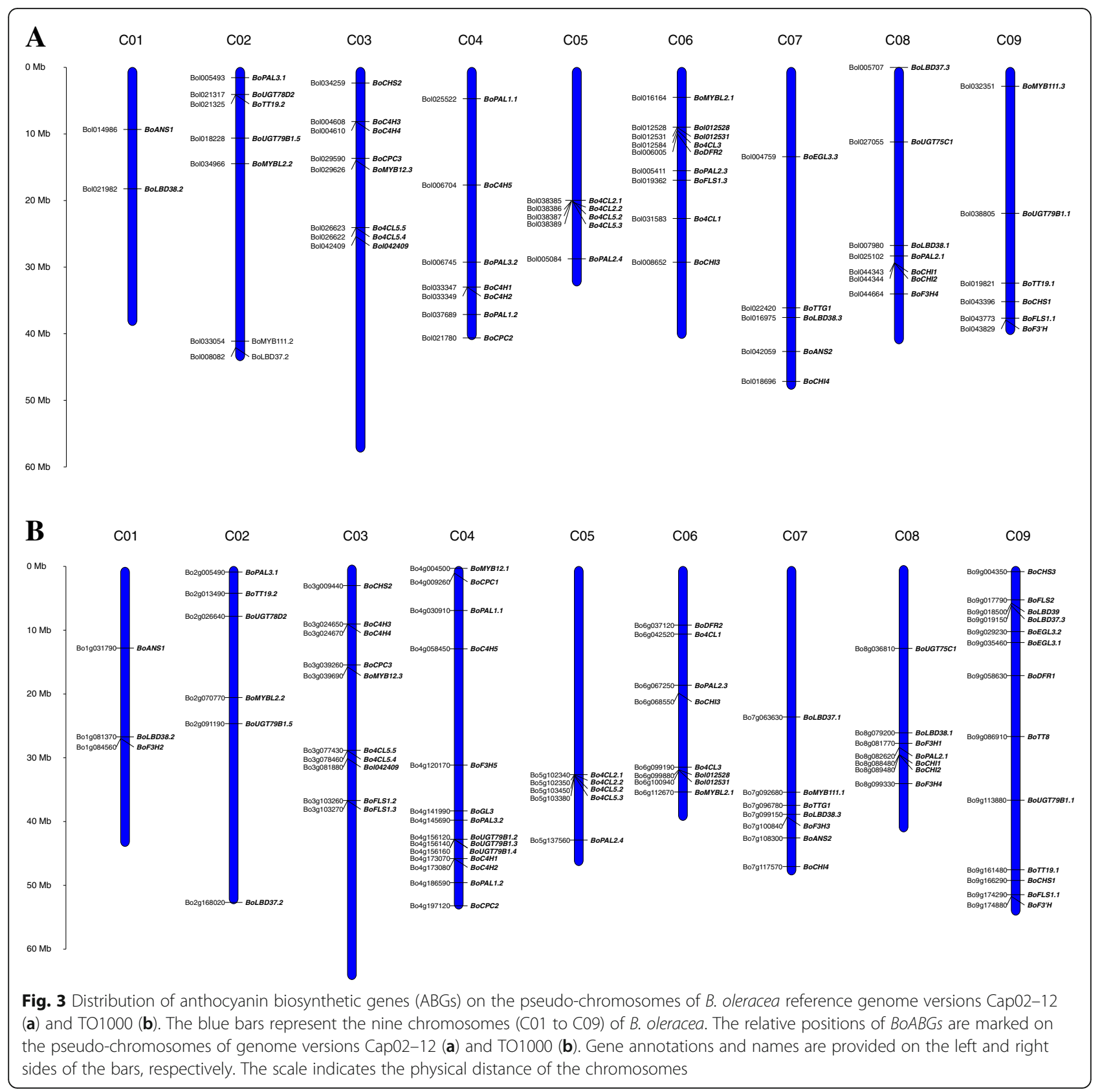

expression level in new leaves of '05-DH-65'; Bol012528 was more highly expressed in '05-DH-65' leaves than in '06-DH-71' leaves, with the highest expression level observed in mature leaves of '05-DH-65' (Fig. 4). Four of the five bHLH coding genes, namely BoGL3, BoEGL3.1, BoEGL3.2, and BoEGL3.3, showed extremely low expression levels in all four leaf types, whereas BoTT8 was more highly expressed in '05-DH65' leaves than in '06-DH-71' leaves. The WD40 protein coding gene, BoTTG1, also showed a higher expression level in '05-DH-65' leaves than in '06-DH-71' leaves (Fig. 4).
The expression patterns of negative regulatory genes in new and mature leaves of '05-DH-65' and '06-DH-71' are shown in Additional file 1: Figure S3. Several transcription factors, including two R3-type single MYB proteins, $M Y B L 2$ and $C P C$, and three $\mathrm{N} / \mathrm{NO}_{3}{ }^{-}$induced members of the $L B D$ gene family, act as negative regulators of anthocyanin biosynthesis in A. thaliana. MYBL2 is a transcriptional repressor that negatively regulates anthocyanin biosynthesis by interacting with TT8 and MBW complexes (Dubos et al., 2008; [22]). BoMYBL2.1 was only expressed in new and mature leaves of '06DH-71'. BoMYBL2.2 was expressed in all four leaf types 


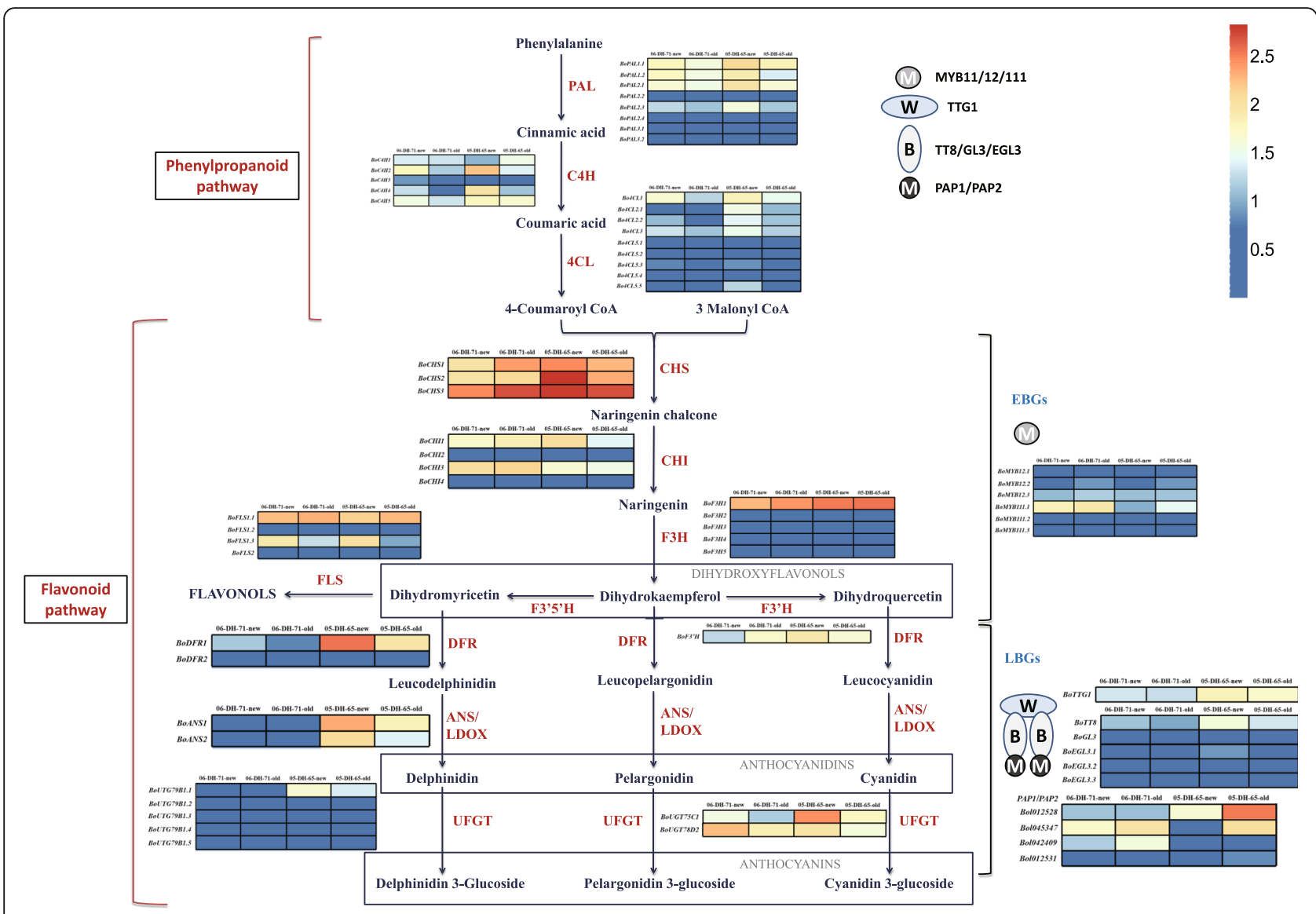

Fig. 4 The anthocyanin biosynthetic pathway and expression levels of structural and positive regulatory genes in B. oleracea. The pathway can be divided into two sections: the phenylpropanoid and the flavonoid pathways. Two types of genes are involved in the flavonoid pathway: early biosynthetic genes (EBGS) and late biosynthetic genes (LBGs). Red type indicates biosynthetic enzymes. The ellipses " $M$ ", " $B$ ", and " $W$ " represent the MYB, bHLH, and WD40 proteins involved in the positive regulation of anthocyanin synthesis. The expression levels of coding genes is indicated by blue and red shading, which represents low to high expression levels, respectively. The color scale corresponds with the mean-centered log2-transformed FPKM values, which are identical to those in Fig. 4

and the expression level was slightly higher in new leaves. CPC, a single-repeat R3-MYB transcription factor, functions as a negative regulator of anthocyanin biosynthesis [43]. BoCPC1, BoCPC2 and BoCPC 3 were all expressed very low in all samples. LBD37, 38, and 39 negatively regulate the late anthocyaninspecific steps by repressing PAP1 and PAP2 under N/ $\mathrm{NO}_{3}{ }^{-}$induction [29]. The BoLBD genes all showed the highest expression levels in new leaves of '05DH-65' and were highly expressed in new leaves than mature leaves.

BoTT19.1 and BoTT19.2, the two TT19 coding genes, were most highly expressed in new leaves of '05-DH-65', second highest in mature leaves of '05-DH-65', followed by new leaves of '06-DH-71', and showed the lowest expression level in mature leaves of '06- $\mathrm{DH}-71$ '. The FPKM value of BoTT19.1 was 380-fold higher in new leaves of '05-DH-65' than in new leaves of '06-DH-71' (Additional file 4: Figure S4).
Based on these results, the expressed levels of genes in anthocyanin biosynthetic pathway from different leaf color samples of ornamental kales were intuitively demonstrated (Fig. 4).

\section{Significant differentially expressed anthocyanin biosynthetic genes between red- and white-leaved accessions}

In order to analyze the significant up- and downregulated BoABGs in red and white leaves of different accessions, the differentially expressed BoABGs were detected in new and mature leaves between red accession '05-DH-65' and white accession '06-DH-71' by software edgeR and DESeq2. The significant differentially expressed genes were selected by the threshold that fold change bigger than 2, $P$ value and FDR (false discovery rate) less than 0.05 for edgeR, as well as fold change bigger than 2 and $q$ value less than 0.05 for DESeq (Additional file 7: Table S4-9). The results of significant 
differentially expression analysis of anthocyanin biosynthetic genes in new and mature leaves between '05-DH65' and '06-DH-71' were listed in Additional file 7: Table S4-9. And the significant up- and down- regulation genes were displayed in two volcano plots by EdgeR analysis (Additional file 5: Figure S5 and Additional file 6: Figure S6). From the results of significant differential genes expression analysis, we found that Bo4CL2.1, BoANS1 and 2, BoDFR1, BoUGT79B1.1, BoTT19.1 and 19.2 were significantly up-regulated both in new and mature leaves of red accession '05-DH-65' by EdgeR and DESeq2 analysis (Fig. 5, Additional file 7: Table S4-9). BoDFR1, BoANS1 and 2, and BoUGT79B1.1 were LBGs leading to biosynthesis anthocyanins. BoTT19.1 and 19.2 were anthocyanin transport genes functioning as carriers which played key roles in anthocyanin transportation and accumulation. The significant up-regulation of these two genes in new and mature leaves of red accession (Fig. 5) might be the essential factor for anthocyanin accumulation in leaves of ornamental kale. For the three MYB genes (Bol012528, Bol012347 and Bol042409) which formed MBW complex activating LBGs, only Bolo12528 was up-regulated in mature leaves of red accession by EdgeR and DESeq2 analysis, while the other two genes were down regulated. BoTT8 and BoTTG1 were both up-regulated in new and mature leaves of '05DH-65' by DESeq2 (Fig. 5). The MYB-bHLH-WD40 protein complex coding genes, Bol012528, BoTT8 and BoTTG1, were up-regulated in red accession might play key positive regulatory roles to active LBGs, such as BoDFR1, BoANS1 and 2. The negative regulation gene $B o M Y B L 2.1$ was significantly down-regulated in new and mature leaves of '05-DH-65' by EdgeR and DESeq2 analysis, indicating its negative regulatory role in anthocyanin biosynthesis of ornamental kale. MYB111.1 and Bol042409 were also down-regulated in new and mature leaves of red accession (Fig. 5), and they might not play as positive regulators of anthocyanin biosynthesis in ornamental kale anymore.

In summary, the significant up-regulation LBGs: BoDFR1, BoANS1 and 2, regulatory genes: Bol012528, BoTT8 and BoTTG1, and transport genes: BoTT19.1 and 19.2 , as well as significant down-regulation negative regulatory gene: BoMYBL2.1, played roles in anthocyanin biosynthesis and accumulation in red ornamental kale.

\section{Discussion}

\section{Variation in leaf color of ornamental kale}

Ornamental kales, which change leaf color from green to red or white under low temperature because of differences in anthocyanin accumulation capabilities, are ideal materials to study the genetic mechanism of anthocyanin biosynthesis of B. oleracea. Anthocyanins were accumulated in the epidermal cells and adjacent mesophyll cells in new leaves of a red-leaved accession, while almost no chlorophylls are present, resulting in the entirely red leaf phenotype. The mature leaves of the red-leaved accession contained a reduced anthocyanins content, but higher amounts of chlorophylls resulting in the green lamina, with only leaf veins and the petiole containing low amounts of chlorophylls exhibiting a light red color. However, the new leaves of the white-leaved accession contained almost no pigments (Fig. 1). The observations showed that the pigment distribution in the different leaves was consistent with the color phenotype, and demonstrated that red pigments were mainly located in the epidermal cells of '05-DH-65' and new leaves contained a higher amount of red pigment than mature leaves. However, almost no pigments were visible in '06-DH-71' new leaves. Thirtyfour anthocyanins were identified in the red-leaved ornamental kale. Cyanidin glycosides are the most common anthocyanins reported in Brassica crop species $[10,18,30]$. It is interesting that one pelargonidin and four delphinidin glycosides were identified, whereas only cyanidin glycosides were detected in Zicaitai (Brassica rapa L. ssp. chinensis var. purpurea) [10], but further verification against standards is needed. The TAC was $\sim 20$-fold higher in new leaves than in mature leaves of the red-leaved accession, whereas almost no anthocyanins were detected in the white-leaved accession (Fig. 2). The present phenotypic assessment and anthocyanins profile and contents analysis illustrated the relationship between the color phenotype and anthocyanin accumulation of the leaves, and provides a foundation to elucidate the genetic mechanism of leaf coloration and anthocyanin biosynthesis in B. oleracea.

\section{Anthocyanin biosynthetic genes expanded by WGD and TD in B. oleracea}

Eighty-one ABGs were identified in the $B$. oleracea genome, which was almost double the number in the $A$. thaliana genome. Whole genome duplication and TD represent the major mechanisms for BoABGs expansion. Some upstream structural genes (phenylpropanoid pathway genes and EBGs) have been expanded through TD to form gene clusters in B. oleracea, such as $\mathrm{C} 4 \mathrm{H}, 4 \mathrm{CL}$, $\mathrm{F} 3 \mathrm{H}$, and FLS coding genes (Table 2). The tandem arrays of $C 4 H, 4 C L$, and $F L S$ are also present in the B. rapa genome [9]. These results demonstrated that upstream structural genes expanded by TD in Brassica species was important for biosynthesis of flavonoid compounds, such as anthocyanins and flavonols. Based on the presence of these homologous structural genes, the anthocyanin biosynthetic pathway in B. oleracea was then established. The regulatory genes were mainly expanded by WGD. Some negative regulators, such as $C P C, L B D 37$, and 


\begin{tabular}{|c|c|c|c|c|}
\hline & \multicolumn{2}{|c|}{ EdgeR } & \multicolumn{2}{|c|}{ DEseq } \\
\hline & New_65_VS_71 & Mature_65_VS_71 & New_65_VS_71 & Mature_65_VS_71 \\
\hline \multicolumn{5}{|l|}{ BoPAL4 } \\
\hline \multicolumn{5}{|l|}{ BoCHS1 } \\
\hline \multicolumn{5}{|l|}{ BoCHS2 } \\
\hline \multicolumn{5}{|l|}{ Bo4CL2.1 } \\
\hline \multicolumn{5}{|l|}{ Bo4CL2.2 } \\
\hline \multicolumn{5}{|l|}{ Bo4CL5.5 } \\
\hline \multicolumn{5}{|l|}{ BoCHII } \\
\hline \multicolumn{5}{|l|}{ ВоСНI3 } \\
\hline \multicolumn{5}{|l|}{ BoC4H1 } \\
\hline \multicolumn{5}{|l|}{$\mathrm{BoC} 4 \mathrm{H} 2$} \\
\hline \multicolumn{5}{|l|}{ BoC4H3 } \\
\hline \multicolumn{5}{|l|}{$\mathrm{BoC} 4 \mathrm{H} 4$} \\
\hline \multicolumn{5}{|l|}{ BoC4H5 } \\
\hline \multicolumn{5}{|l|}{$\mathrm{BoF}^{\prime} \mathrm{H}$} \\
\hline \multicolumn{5}{|l|}{ BoFLS1.1 } \\
\hline \multicolumn{5}{|l|}{ BoDFR1 } \\
\hline \multicolumn{5}{|l|}{ BoANS1 } \\
\hline \multicolumn{5}{|l|}{ BoANS2 } \\
\hline \multicolumn{5}{|l|}{ BoUGT79B1.1 } \\
\hline \multicolumn{5}{|l|}{ BoUGT75Cl } \\
\hline \multicolumn{5}{|l|}{ BoUGT78D2 } \\
\hline \multicolumn{5}{|l|}{ BoTT19.1 } \\
\hline \multicolumn{5}{|l|}{ BoTT19.2 } \\
\hline \multicolumn{5}{|l|}{ BoMYB12.1 } \\
\hline \multicolumn{5}{|l|}{ MYB111.1 } \\
\hline \multicolumn{5}{|l|}{ Bol012528 } \\
\hline \multicolumn{5}{|l|}{ Bol045347 } \\
\hline Bol042409 & & & & \\
\hline BoTT8 & & & & \\
\hline BoEGL3.1 & & & & \\
\hline BoTTGI & & & & \\
\hline BoMYBL2.1 & & & & \\
\hline BoMYBL2.2 & & & & \\
\hline BoLBD39 & & & & \\
\hline
\end{tabular}

Fig. 5 The significant differential expression ABGs in new and mature leaves between red accession '05-DH-65' and white accession '06-DH-71' by EdgeR and DEseq analysis. The red and green colors indicated significant up- and down-regulation of genes in new and mature leaves of '05-DH65 , and the gray ones showed on significant expression difference

$L B D 38$, retained three copies in different subgenomes (Table 2). From the present expression data, we speculate that these negative regulators might not play important negative regulatory roles in anthocyanin biosynthesis of ornamental kale (Additional file 3: Figure S3). These genes might have undergone neo- or subfunctionalization after WGD. The distribution analysis showed that some BoABGs were mapped to different locations of the two $B$. oleracea reference genome versions (Fig. 3). A higher number of BoABGs were mapped on the genome V2.1 (TO1000), which indicated that the assembly of TO1000 was more complete. The differences in distribution of BoABGs might also reflect assembly errors or structural variations of the two reference 
genome sequences, so additional complete and accurate reference genomic and pan-genomic sequences are essential for $B$. oleracea. The distribution analysis may provide a basis for further genetic mapping and molecular breeding studies. Identification of the complete set of anthocyanin biosynthetic genes in the B. oleracea genome provides a valuable resource for investigations of genetic mechanisms.

\section{Differential expression of BoABGs determines anthocyanin accumulation in ornamental kales}

The higher expression levels of upstream biosynthetic genes in new leaves of the ornamental kale accessions provides the capacity to synthesize higher quantities of flavonoid compounds, such as anthocyanins and flavonols. In leaves, flavonoids are usually considered to function as antioxidants [38]. We present the following hypotheses. Under low temperature, flavonoid biosynthesis might be induced to accumulate flavonoid compounds in ornamental kale to protect the new leaves from intracellular damage caused by enhanced accumulation of reactive oxygen species. Given the genetic variation, different genotypes would synthesize and accumulate different flavonoids; thus, red-leaved accessions may accumulate anthocyanins whereas colorless flavonoids would accumulate in white-leaved accessions that are unable to synthesize anthocyanins. The LBGs leading to anthocyanins biosynthesis, such as BoDFR1, BoANS1 and 2, and BoUGT79B1.1, were most highly expressed in the new leaves of the red-leaved accession, and second highest in mature leaves of the red-leaved accession, and showed an extremely low expression level in the leaves of the white-leaved accession. The expression patterns of these LBGs in the different leaf types were in accordance with the anthocyanins contents and leaf color phenotypes. These genes may play vital roles in anthocyanin biosynthesis in ornamental kale.

The positive regulatory genes Bol012528, BoTT8, and BoTTG1, which form the MBW transcription complex to activate LBGs, such as BoDFR1 and BoANS1 and 2, positively regulate anthocyanins biosynthesis in ornamental kale. The negative regulatory gene BoMYBL2.1 was only expressed in leaves of the white-leaved accession '06-DH-71', which would repress LBGs expression in white and green leaves to prevent anthocyanins biosynthesis. Other negative regulatory genes, such as BoLBDs, which showed the higher expression levels in new leaves, did not play negative roles in anthocyanin biosynthesis of ornamental kale. BoMYBL2.1 was indicated to play the main negative regulatory role in anthocyanin accumulation in ornamental kale.

TT19 is a carrier protein for sequestering anthocyanin into the vacuole. The TT19 mutant of Arabidopsis with no visible anthocyanins accumulated in cotyledons and hypocotyls of seedlings grown on the anthocyanin induction media demonstrated the key role of TT19 in anthocyanin accumulation and coloration [32]. BoTT19.1 and BoTT19.2 showed a significantly higher expression level in red leaves than in white and green leaves. The expression patterns of these genes were consistent with the patterns of anthocyanins accumulation and color phenotypes of different leaves of red- and white-leaved ornamental kale accessions. The DEGs analysis also demonstrated that BoANS1 and 2, BoDFR1, BoUGT79B1.1, BoTT19.1 and 19.2 were significantly up-regulated in red accession, while BoMYBL2.1 was significantly up-regulated in white accession (Fig. 5). Thus, these genes are indicated to play vital roles in anthocyanins biosynthesis and accumulation in ornamental kale.

\section{Genetic network of anthocyanins accumulation in ornamental kale}

Under low temperature, the new leaves of different ornamental kale genotypes will exhibit red and white colors because of inhibition of chlorophyll synthesis and variation in anthocyanins accumulation capacities. To elucidate the genetic mechanism of anthocyanin biosynthesis and leaf color variation of ornamental kales, a comparative whole-genome identification and expression analysis of anthocyanins between red- and white-leaved accessions of BoABGs was conducted. Based on these results, a metabolic pathway and regulatory network was constructed to represent anthocyanins biosynthesis and accumulation in ornamental kales (Fig. 6). The positive regulatory genes Bol012528, BoTT8, and BoTTG1 forming the MBW complex were up-regulated in the red-leaved accession to activate the LBGs, especially BoDFR1, BoANS1 and 2, and BoUGT79B1.1, which lead to anthocyanins synthesis. In contrast, these positive regulatory genes were down-regulated and a negative regulatory gene, BoMYBL2.1, was up-regulated in the white and green leaves of the white-leaved accession, thereby repressing the LBGs to inhibit anthocyanin biosynthesis. The three MBW complex-forming positive regulatory genes and one negative regulatory gene functioned as transcriptional factors to regulate anthocyanin biosynthesis in ornamental kale. The anthocyanins transport genes BoTT19.1 and 19.2, which were almost only expressed in red leaves, would play vital roles in anthocyanins accumulation in ornamental kale. This genetic regulatory network of anthocyanins accumulation in different accessions explains the variation in leaf color and enhances the understanding of leaf color development under low temperature in ornamental kale. These findings pave the way for further genetic modification of leaf color and the breeding of novel cultivars of $B$. oleracea. 


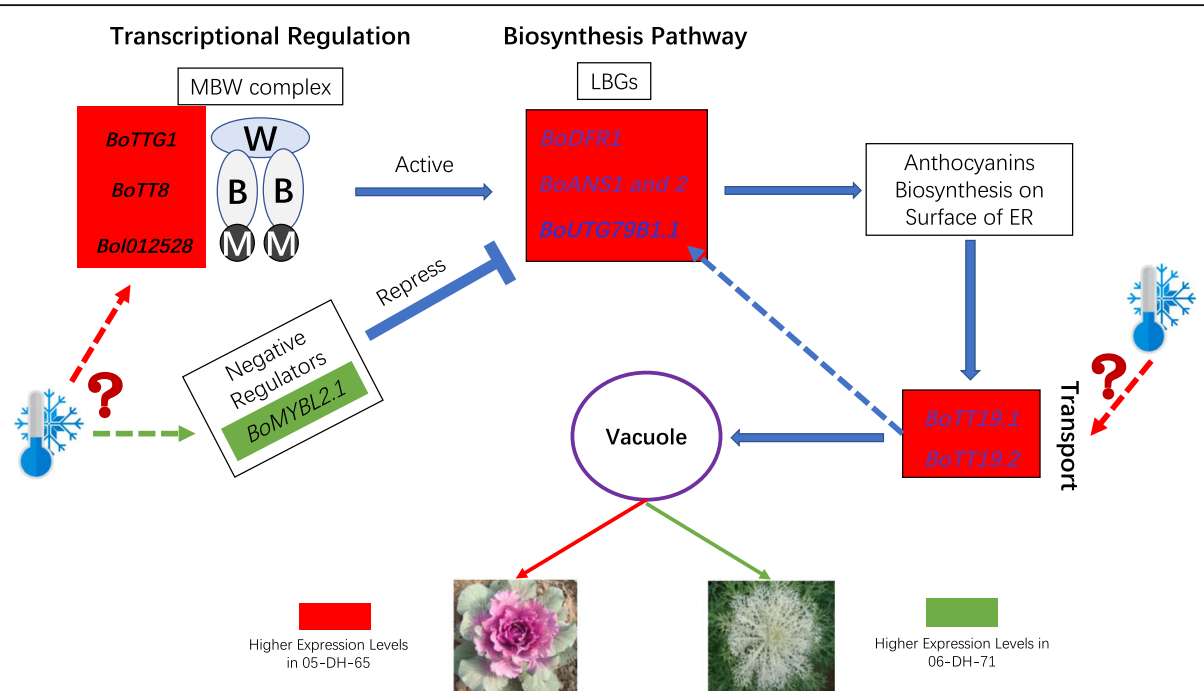

Fig. 6 Regulatory network of anthocyanins accumulation in red- and white-leaved ornamental kales. In red leaves, BOTTG1, BOTT8, and Bol012528 form the MBW complex to activate BODFR1, BOANS1 and 2, BOUGT79B1.1, and BOUT78D2 to promote anthocyanins biosynthesis on the cytosolic surface of the ER. In white and green leaves, the negative regulator BOMYBL2.1 is highly expressed to repress the LBGs. The transporter genes BoTT19.1 and 19.2 are highly expressed in red leaves to transport anthocyanins from the ER to the vacuole, thereby promoting the red leaf phenotype. High expression levels of BoTT19.1 and 19.2 may also feedback to activate LBGs by transporting greater amounts of anthocyanins

\section{Conclusions}

We determined pigments distributions in red- and white-leaved accessions which could explain the leaf color phenotypes of ornamental kales. Thirty-four anthocyanins were detected in the red-leaved accession. Eighty-one anthocyanin biosynthetic genes were identified in the B. oleracea reference genome. The expression patterns of these genes in different leaf types indicated that late biosynthetic genes (BoDFR1, BoANS1 and 2 and BoUGT79B1.1), positive regulatory genes (BoTTG1, BoTT8, and Bol012528), a negative regulatory gene (BoMYBL2.1), and transport genes (BoTT19.1 and BoTT19.2) play key roles in anthocyanin accumulation in ornamental kale. A genetic regulatory network of anthocyanin accumulation in ornamental kale was constructed. These results enhance the understanding of the genetic mechanisms and regulatory network of anthocyanin accumulation in B. oleracea under low temperature, and provide a theoretical basis for breeding new cultivars of Brassica vegetables with increased anthocyanin contents and cold resistance.

\section{Methods}

Plant materials, phenotypic characterization, and leaf anatomical observations

Two ornamental kale doubled-haploid (DH) lines (produced by vegetable biotechnology group, Beijing Vegetable Resarch Center), '05-DH-65' and '06-DH-71', which differ in leaf color and shape, were selected for phenotype assessment, anthocyanins profiling, and RNA-seq analysis. '05-DH-65' has entire leaves and the central leaves are red under low temperature (Fig. 1a), whereas '06-DH-71' has feather-like divided leaves and the central leaves change in color from green to white under low temperature (Fig. 1b). The plant materials were grown in an unheated research greenhouse of the $\mathrm{Na}$ tional Engineering Research Center for Vegetables, Beijing, China, during the 2016-2017 cropping season. The two DH lines were cultivated side by side each with three replications. Central (young) and outer (mature) leaves were collected at the rosette stage after color development (at temperatures under $10{ }^{\circ} \mathrm{C}$ ) for observation of leaf anatomy, analysis of anthocyanin contents and profiles, and RNA extraction for RNA-seq analysis. The phenotype of the central and outer leaves from '05-DH65 ' and '06-DH-71' was compared. In addition, to examine purple pigment accumulation, portions of the lamina and petiole were transversely and longitudinally sectioned, respectively, by hand and examined with a Nikon TS100 microscope.

\section{Anthocyanins content determination by $\mathrm{pH}$ differential spectrophotometry}

The $\mathrm{pH}$ differential spectrophotometry method was performed in accordance to that reported by [15], with slight modifications [10], to determine total anthocyanins content (TAC) in central and outer leaves of '05DH-65' and '06-DH-71'. Absorbance was measured at 520 and $700 \mathrm{~nm}$. The TAC was expressed as cyanidin-3glucoside (cyd-glu, molar extinction coefficient 26,900 l/ $\mathrm{cm}$ mol, molecular weight $449.2 \mathrm{~g} / \mathrm{mol}$ ) equivalents. The units for TAC were $\mathrm{mg} / \mathrm{g}$ dry weight (DW) of the 
detected sample. For all samples, TAC measurements were replicated three times.

\section{Anthocyanin profile characterization}

The chromatographic system, which was set up in accordance with the method described previously [10] with modifications, which consisted of a Shimadzu LC-30A UHPLC system equipped with an ultraviolet detector. Chromatographic separation was achieved using a Waters ACQUITY UPLC ${ }^{\mathrm{mm}}$ BEH C18 $(2.1 \times 100 \mathrm{~mm}, 1.7 \mu \mathrm{m})$ at a flow rate of $0.3 \mathrm{ml} / \mathrm{min}$. The column oven temperature was set at $40^{\circ} \mathrm{C}$. The mobile phase was a binary solvent system consisting of solvent A (formic acid $0.1 \%[\mathrm{v} / \mathrm{v}$ ] in water) and solvent $\mathrm{B}$ (acetonitrile). The gradient conditions were as follows: $5-15 \% \mathrm{~B}$ from 0 to $25 \mathrm{~min}$, maintained at $15 \%$ B for $20 \mathrm{~min}$; $15-18 \%$ B from 45 to $60 \mathrm{~min} ; 18-95 \%$ B from 60 to $70 \mathrm{~min}$, maintained at 95\% B for $2 \mathrm{~min}$; and 5\% B from 72 to $75 \mathrm{~min}$ for equilibration of the column for the next run. The sample injection volume was $3 \mu \mathrm{L}$. Chromatograms were recorded at $520 \mathrm{~nm}$ for anthocyanins. The analytes were identified using an AB SCIEX TripleTOF 6600 system equipped with a Turbo $\mathrm{V}^{\mathrm{sm}}$ ion source. Product ion spectra were acquired using time-of-flight mass spectrometry and information-dependent acquisition compound scan modes. The data acquisition used Analyst ${ }^{\circ}$ TF 1.7.1 software, and PeakView ${ }^{\circ} 2.1$ and MasterView ${ }^{\text {Tx }}$ software were used for data analysis.

\section{Identification of anthocyanin biosynthetic genes by comparative genomic analysis}

Anthocyanin biosynthetic genes of B. oleracea were identified by syntenic and non-syntenic homolog analysis with $A$. thaliana. The reference genome of B. oleracea we used for anthocyanin biosynthetic genes identification was Cap02-12 [19]. We identified syntenic orthologs between A. thaliana and B. oleracea from the Brassica Database (BRAD; http://brassicadb.org/brad/) using both sequence similarities (cutoff: $\mathrm{E} \leq 1 \mathrm{E}-20$ ) and the collinearity levels of flanking genes [7]. The homologous relationships were analyzed by BLASTN and BLASTP algorithm-based searches using gene and protein sequences, respectively, with a cut off E-value $\leq 1 \mathrm{E}-10$ and coverage $\geq 0.75$ [9]. Then we identified the homolog genes in another reference genome TO1000 [24], and analyzed the chromosomes locations of these genes on the two references.

\section{RNA extraction}

Total RNA was extracted using TRIzol ${ }^{\mathrm{Tm}}$ reagent (Invitrogen, 15,596-026). The contaminant DNA was removed using the TURBO DNA-free ${ }^{\mathrm{Tw}}$ Kit (Ambion, AM1907). The quality of purified RNA was initially evaluated on agarose gel and then quantified using a NanoDrop ${ }^{\text {tx }}$ spectrophotometer (Thermo Fisher Scientific, Inc.). The integrity of RNA samples was further evaluated using an Agilent 2100 Bioanalyzer (Agilent Technologies, Inc.).

\section{Library construction and sequencing}

The TruSeq ${ }^{\text {Tu }}$ RNA Sample Preparation Kit (Illumina, Inc.) was used to construct cDNA libraries in accordance with the manufacturer's instructions. Briefly, poly-A mRNA was purified and fragmented into short fragments and used as templates for first-strand cDNA synthesis. DNA polymerase $\mathrm{I}$ and RNase $\mathrm{H}$ were used to synthesize the secondstrand cDNA. Purified short double-strand cDNA fragments were connected with adapters (Illumina). Suitable ligated cDNA fragments were selected as templates for PCR amplification for final library construction. Finally, the cDNA libraries were sequenced using the Illumina HiSeq ${ }^{\circ}$ 2500 platform.

\section{RNA-seq data analysis}

Adaptors were removed from the RNA-Seq reads. The reads in which unknown bases comprised more than 5\% of the total and low-quality reads (the percentage of the low-quality bases of quality value $\leq 5$ is more than $50 \%$ in a read) were also removed. The clean reads were aligned to the cabbage genome (B. oleracea var. capitata line $02-$ 12) [19] accessed from BRAD [5], allowing up to two mismatches in each segment alignment by TopHat [34, 35] and Bowtie software. Only unique mapped reads were used for further analysis.

Gene expression level and transcript abundances were calculated using the FPKM (fragments per kilobase of transcript per million mapped reads) method [36]. The FPKM values of anthocyanin biosynthetic genes from leaves of the different ornamental kales were $\log 2$ transformed (in order to avoid the minus value after transformation, we added "1" to every FPKM values before $\log 2$ transformation), mean centered, and further used for hierarchical clustering and heatmap generation with the pheatmap package in $R$ software.

\section{Differential gene expression analysis}

The differential expression analysis was carried out by using the Bioconductor package EdgeR (Empirical Analysis of Digital Gene Expression Data in R) (Robinson et al., 2010 and McCarthy et al., 2012) and DESeq2 package [1]. The significant differential expression genes were selected by the threshold that fold change bigger than 2, $\mathrm{P}_{-}$value and FDR (false discovery rate) less than 0.05 .

\section{Additional files}

Additional file 1: Figure S1. (A) The expression values distribution of different samples demonstrated the overall expression levels among different samples. (B) A cluster dendrogram showed biological replicates 
sampled with good agreement replicates which was analyzed by gene expression values between each other. (PDF $813 \mathrm{~kb}$ )

Additional file 2: Figure S2. Heatmap representing expression profiles of anthocyanin biosynthetic genes in new and mature leaves of the ornamental kale $\mathrm{DH}$ lines '05-DH-65' and '06-DH-71'. Blue and red colors are used to represent low to high expression levels, respectively. The color scale corresponds to the mean-centered log2-transformed FPKM values. (PDF $190 \mathrm{~kb}$ )

Additional file 3: Figure S3. Expression levels of negative regulatory genes involved in anthocyanin biosynthesis in new and mature leaves of the ornamental kale DH lines '05-DH-65' and '06-DH-71'. (PDF 13 kb)

Additional file 4: Figure S4. Expression levels of the transport genes TT19.1 and TT19.2 in new and mature leaves of the ornamental kale DH lines '05-DH-71' and '06-DH-71'. (PDF $252 \mathrm{~kb}$ )

Additional file 5: Figure S5. Volcano plot demonstrated significantly expressed BoABGs between new leaves of '05-DH-65' and '06-DH-71' by edgeR DEGs analysis. Green dots indicated the eight significantly upregulated while red dots indicated the 11 significantly down-regulated BoABGs in new leaves of '05-DH-65'. (PDF $271 \mathrm{~kb}$ )

Additional file 6: Figure S6. Volcano plot demonstrated significantly expressed BoABGs between mature leaves of ' $05-\mathrm{DH}-65^{\prime}$ and '06-DH-71 by edgeR DEGs analysis. Green dots indicated the nine significantly upregulated while red dots indicated the six significantly down-regulated BoABGs in mature leaves of '05-DH-65'. (PDF $230 \mathrm{~kb}$ )

Additional file 7: Table S1. The corresponding gene IDs of ABGs in the two reference genome versions of $B$. oleracea. Table S2. The FPKM values of ABGs in red- and white- leaf color accessions. (XLSX $77 \mathrm{~kb}$ )

\section{Abbreviations}

4CL: 4-coumarate: CoA ligase; ABGs: Anthocyanin biosynthetic genes; ANS: Anthocyanidin synthase; AtABGs: Anthocyanin biosynthetic genes in A. thaliana; bHLH: Basic helix-loop-helix; BoABGs: Anthocyanin biosynthetic genes in B. oleracea; BrABGs: Anthocyanin biosynthetic genes in B. rapa; C4H: Cinnamate-4-hydroxylase; $\mathrm{CHI}$ : Chalcone isomerase; CHS: Chalcone synthase; CPC: CAPRICE; DFR: Dihydroflavonol-4-reductase; EBGs: Early biosynthetic genes; EGL3: Enhancer of glabrous 3; ER: Endoplasmic reticulum; F3'H: Flavonoid 3'-hydroxylase; F3H: Flavanone 3-hydroxylase; FLS: Flavonol synthase; FPKM: Fragments per kilobase of transcript per million mapped reads; GL3: Glabrous 3; GST: Glutathione S-transferase; LBD: Lateral organ boundary domain; LBGs: Late biosynthetic genes; LC-MS: Liquid chromatography-mass spectrometry; MBW: MYB-bHLH-WD40; MYBL2: MYBlike 2; PAL: Phenylalanine ammonia-lyase; PAP1/2: Production of anthocyanin pigment 1/2; TAC: total anthocyanins content; TD: Tandem duplication; TT8/ 19: Transparent testa 8/19; TTG1: Transparent testa glabrous 1; UFGT: UDPglucose:flavonoid 3-O-glucosyltransferase; WGD: Whole genome duplication; WGT: Whole genome triplication

\section{Acknowledgements}

Research was carried out in the Key Laboratory of Biology and Genetic Improvement of Horticultural Crops (North China), Ministry of Agriculture, P. R. China.

We thank Robert McKenzie, PhD, from Liwen Bianji, Edanz Group China (http://www.liwenbianji.cn/ac), for editing the English text of a draft of this manuscript.

\section{Author contributions statement}

NG designed the experiments, performed the RNA-seq data analysis and regulatory network construction, and drafted the manuscript. SH grew the plant materials, conducted the phenotypes assessment, and performed anatomical observations. GW identified anthocyanin biosynthetic genes in $B$. oleracea. MZ extracted RNAs and determined anthocyanin contents. SZ performed anthocyanin profile and content analysis. FL conceived and supervised the work, and modified the manuscript. All of the authors have read and approved the final manuscript.

\section{Consent for publication}

Not Applicable.

\section{Funding}

The Science and Technology Innovation Capacity Building Projects of the Beijing Academy of Agriculture and Forestry Sciences (KJCX20170413); National Natural Science Foundation of China (NSFC grant: 31501752); The overseas training program of the State Administration of Foreign Experts Affairs of China (P172001038):

The National Spark Program Projects of China (2015GA600004);

The International Cooperation Fund of the Beijing Academy of Agriculture and Forestry Sciences (GJHZ2017-2)

These funding bodies provided financial supports for this study.

\section{Availability of data and materials}

The plant materials as well as the raw data of their phenotypes and anthocyanin profiles during the current study are available from the corresponding author on reasonable request.

The reference genome of B. oleracea Cap02-12 and TO1000 are available from Brassca Database (BRAD): http://brassicadb.org/brad/ and EnsemblPlants genome database: http://plants.ensembl.org/Brassica oleracea/Info/Index, respectively.

The RNA-seq datasets during the current study are available from the corresponding author on reasonable request.

The expression datasets of antocyanin biosynthetic genes in red- and whiteleaved ornamental kales are included in this published article.

\section{Ethics approval and consent to participate}

Not Applicable.

\section{Competing interests}

All of the authors declare that there are no personal, professional or financial relationships that could potentially be construed as a conflict of interest.

\section{Author details}

'Beijing Vegetable Research Center, Beijing Academy of Agriculture and Forestry Sciences, National Engineering Research Center for Vegetables, Key Laboratory of Biology and Genetic Improvement of Horticultural Crops (North China), Ministry of Agriculture, P. R. China, Beijing 100097, China ${ }^{2}$ Institute of Vegetables and Flowers, Chinese Academy of Agricultural Sciences, Beijing 100081, China.

Received: 31 October 2018 Accepted: 17 June 2019

Published online: 08 July 2019

\section{References}

1. Anders S, Huber W. Differential expression of RNA-Seq data at the gene level-the DESeq package. Heidelberg, Germany: European Molecular Biology Laboratory (EMBL); 2012.

2. Borevitz JO, Xia Y, Blount J, Dixon RA, Lamb C. Activation tagging identifies a conserved MYB regulator of phenylpropanoid biosynthesis. Plant Cell. 2000;12(12):2383-94.

3. Broun P. Transcriptional control of flavonoid biosynthesis: a complex network of conserved regulators involved in multiple aspects of differentiation in Arabidopsis. Curr Opin Plant Biol. 2005;8(3):272-9.

4. Butelli E, Titta L, Giorgio M, Mock HP, Matros A, Peterek S, et al. Enrichment of tomato fruit with health-promoting anthocyanins by expression of select transcription factors. Nat Biotechnol. 2008;26(11):1301-8.

5. Cheng F, Liu S, Wu J, Fang L, Sun S, Liu B, et al. BRAD, the genetics and genomics database for Brassica plants. BMC Plant Biol. 2011;11(1):13

6. Dubos C, Le GJ, Baudry A, Huep G, Lanet E, Debeaujon I, et al. MYBL2 is a new regulator of flavonoid biosynthesis in Arabidopsis thaliana. Plant J. 2008;55(6):940-53.

7. Feng C, Jian W, Lu F, Wang X. Syntenic gene analysis between Brassica rapa and other Brassicaceae species. Front Plant Sci. 2012:3(198):198.

8. Gonzalez A, Zhao M, Leavitt JM, Lloyd AM. Regulation of the anthocyanin biosynthetic pathway by the TG1/bHLH/Myb transcriptional complex in Arabidopsis seedlings. Plant J. 2008:53(5):814-27.

9. Guo N, Cheng F, Wu J, Liu B, Zheng S, Liang J, et al. Anthocyanin biosynthetic genes in Brassica rapa. BMC Genomics. 2014;487(1):135-42.

10. Guo N, Wu J, Zheng S, Cheng F, Liu B, Liang J, et al. Anthocyanin profile characterization and quantitative trait locus mapping in zicaitai ( Brassica rapa L. ssp. chinensis var. purpurea ). Mol Breed. 2015;35(5):113. 
11. Hichri I, Barrieu F, Bogs J, Kappel C, Delrot S, Lauvergeat V. Recent advances in the transcriptional regulation of the flavonoid biosynthetic pathway. J Exp Bot. 2011;62(8):2465.

12. Holton TA, Cornish EC. Genetics and biochemistry of anthocyanin biosynthesis. Plant Cell. 1995;7(7):1071-83.

13. Hou DX. Potential mechanisms of cancer chemoprevention by anthocyanins. Curr Mol Med. 2003;3(2):149

14. Koes R, Verweij W, Quattrocchio F. Flavonoids: a colorful model for the regulation and evolution of biochemical pathways. Trends Plant Sci. 2005; 10(5):236-42.

15. Lee J, Durst RW, Wrolstad RE. Determination of total monomeric anthocyanin pigment content of fruit juices, beverages, natural colorants, and wines by the $\mathrm{pH}$ differential method: collaborative study. J AOAC Int. 2005;88(5):1269.

16. Lepiniec L, Debeaujon I, Routaboul JM, Baudry A, Pourcel L, Nesi N, et al. Genetics and biochemistry of seed flavonoids. Annu Rev Plant Biol. 2006; 57(1):405-30.

17. Li Y, Yu X. Pollination with laser-irradiated pollens breaks crossincompatibility between zicaitai (Brassica campestris var. purpurea) and ornamental kale (Brassica oleracea var. acephala) to produce hybrids with the aid of ovule culture. Sci Hortic. 2006;108(4):397-402.

18. Lin LZ, Sun J, Chen P, Harnly J. UHPLC-PDA-ESI/HRMS/MSn analysis of anthocyanins, Flavonol glycosides, and Hydroxycinnamic acid derivatives in red mustard greens (Brassica juncea Coss variety). J Agric Food Chem. 2011; 59(22).

19. Liu S, Liu Y, Yang X, Tong C, Edwards D, Parkin IAP, et al. The Brassica oleracea genome reveals the asymmetrical evolution of polyploidgenomes. Nat Commun. 2014;5(5):3930.

20. Liu XP, Gao BZ, Han FQ, Fang ZY, Yang LM, Zhuang M, et al. Genetics and fine mapping of a purple leaf gene, BoPr, in ornamental kale (Brassica oleracea L. var. acephala ). BMC Genomics. 2017;18(1):230

21. Martin C, Butelli E, Petroni $K$, Tonelli C. How can research on plants contribute to promoting human health? Plant Cell. 2011;23(5):1685-99.

22. Matsui K, Umemura Y, Ohme-Takagi M. AtMYBL2, a protein with a single MYB domain, acts as a negative regulator of anthocyanin biosynthesis in Arabidopsis. Plant J. 2008:55(6):954-67.

23. Nesi N, Debeaujon I, Jond C, Pelletier G, Caboche M, Lepiniec L. The TT8 gene encodes a basic helix-loop-helix domain protein required for expression of DFR and BAN genes in Arabidopsis siliques. Plant Cell. 2000; 12(10):1863.

24. Parkin IA, Koh C, Tang H, Robinson SJ, Kagale S, Clarke WE, et al. Transcriptome and methylome profiling reveals relics of genome dominance in the mesopolyploid Brassica oleracea. Genome Biol. 2014;15(6): R77.

25. Payne CT, Zhang F, Lloyd AM. GL3 encodes a bHLH protein that regulates trichome development in Arabidopsis through interaction with GL1 and TTG1. Genetics. 2000;156(3):1349.

26. Petroni $\mathrm{K}$, Tonelli $\mathrm{C}$. Recent advances on the regulation of anthocyanin synthesis in reproductive organs. Plant Sci. 2011;181(3):219-29.

27. Podsędek A. Natural antioxidants and antioxidant capacity of Brassica vegetables: a review. LWT Food Sci Technol. 2007;40(1):1-11.

28. Ren J, Liu Z, Niu R, Feng H. Mapping of Re, a gene conferring the red leaf trait in ornamental kale (Brassica oleracea L. var. acephala). Plant Breed. 2015; 134(4):494-500.

29. Rubin G, Tohge T, Matsuda F, Saito K, Scheible W-R. Members of the LBD family of transcription factors repress anthocyanin synthesis and affect additional nitrogen responses in Arabidopsis. The Plant Cell Online. 2009: 21(11):3567-84

30. Scalzo RL, Genna A, Branca F, Chedin M, Chassaigne H. Anthocyanin composition of cauliflower (Brassica oleracea L. var. botrytis) and cabbage (B. oleracea L. var. capitata) and its stability in relation to thermal treatments. Food Chem. 2008;107(1):136-44.

31. Stracke R, Jahns O, Keck M, Tohge T, Niehaus K, Fernie AR, et al. Analysis of PRODUCTION OF FLAVONOL GLYCOSIDES-dependent flavonol glycoside accumulation in Arabidopsis thaliana plants reveals MYB11-, MYB12-and MYB111-independent flavonol glycoside accumulation. New Phytol. 2010; 188(4):985-1000

32. Sun Y, Li H, Huang J-R. Arabidopsis TT19 functions as a carrier to transport anthocyanin from the cytosol to tonoplasts. Mol Plant. 2012;5(2):387-400.

33. Tanaka Y, Ohmiya A. Seeing is believing: engineering anthocyanin and carotenoid biosynthetic pathways. Curr Opin Biotechnol. 2008;19(2):190-7.
34. Trapnell C, Pachter L, Salzberg SL. TopHat: discovering splice junctions with RNA-Seq. Bioinformatics. 2009:25(9):1105-11.

35. Trapnell C, Williams BA, Pertea G, Mortazavi A, Kwan G, van Baren MJ, et al. Transcript assembly and quantification by RNA-Seq reveals unannotated transcripts and isoform switching during cell differentiation. Nat Biotechnol. 2010;28(5):511-5.

36. Wagner GP, Kin K, Lynch VJ. Measurement of mRNA abundance using RNAseq data: RPKM measure is inconsistent among samples. Theory Biosci. 2012;131(4):281-5.

37. Walker AR, Davison PA, Bolognesi-Winfield AC, James $C M$, Srinivasan $N$, Blundell TL, et al. The TRANSPARENT TESTA GLABRA1 locus, which regulates trichome differentiation and anthocyanin biosynthesis in Arabidopsis, encodes a WD40 repeat protein. Plant Cell. 1999:11(7):1337-50.

38. Winkelshirley B. Flavonoid biosynthesis. A colorful model for genetics, biochemistry, cell biology, and biotechnology. Plant Physiol. 2001;126(2): 485-93.

39. Wu X, Prior RL. Systematic identification and characterization of anthocyanins by HPLC-ESI-MS/MS in common foods in the United States: fruits and berries. J Agric Food Chem. 2005;53(7):2589-99.

40. Xu, Wenjia, Dubos, Christian, Lepiniec, and Loïc (2015). Transcriptional control of flavonoid biosynthesis by MYB-bHLH-WDR complexes Trends in Plant Science 20(3), 176-185.

41. Xu, W., Grain, D., Le, G.J., Harscoët, E., Berger, A., Jauvion, V., et al. (2013). Regulation of flavonoid biosynthesis involves an unexpected complex transcriptional regulation of TT8 expression, in Arabidopsis New Phytologist 198(1), 59-70

42. Zhang F, Gonzalez A, Zhao M, Payne CT, Lloyd A. A network of redundant bHLH proteins functions in all TTG1-dependent pathways of Arabidopsis. Development. 2003;130(20):4859-69.

43. Zhu HF, Fitzsimmons K, Khandelwal A, Kranz RG. CPC, a single-repeat R3 $M Y B$, is a negative regulator of anthocyanin biosynthesis in Arabidopsis. Mol Plant. 2009:2(4):790-802.

44. Zhu P, Cheng M, Feng X, Xiong Y, Liu C, Kang Y. Mapping of pi , a gene conferring pink leaf in ornamental kale (Brassica oleracea L. var. Acephala DC). Euphytica. 2016;207(2):377-85.

\section{Publisher's Note}

Springer Nature remains neutral with regard to jurisdictional claims in published maps and institutional affiliations.
Ready to submit your research? Choose BMC and benefit from:

- fast, convenient online submission

- thorough peer review by experienced researchers in your field

- rapid publication on acceptance

- support for research data, including large and complex data types

- gold Open Access which fosters wider collaboration and increased citations

- maximum visibility for your research: over $100 \mathrm{M}$ website views per year

At $\mathrm{BMC}$, research is always in progress.

Learn more biomedcentral.com/submissions 